\title{
Identification of grapevine accessions from Argentina introduced in the ampelographic collection of Domaine de Vassal
}

\author{
Jean-Michel Boursiquot ${ }^{1}$, Valérie Laucou ${ }^{1}$, Alcides Llorente ${ }^{2}$ and Thierry Lacombe ${ }^{1}$ \\ ${ }^{1}$ Montpellier SupAgro - INRA, UMR AGAP, Equipe Diversité, Adaptation et Amélioration de la Vigne, Place Viala, \\ 34060 Montpellier Cedex, France \\ ${ }^{2}$ General Roca, Rio Negro, Argentina
}

\begin{abstract}
The study of accessions from Argentina may provide a valuable testimony on the origins of the different genetic resources and varieties which were sought and used to develop the vineyard of this country. 54 accessions introduced in the Vassal collection were analyzed using 20 microsatellite markers and ampelographic descriptors. Among them, 43 distinct varieties have been identified: 22 correspond to old traditional wine or table grape varieties from Europe and Middle East, 12 are probably varieties selected in Argentina or South America and are mostly descendants of Muscat of Alexandria and Mission, 4 are seedlings obtained in Italy, USA and Bulgaria, 2 are specific mutants and 3 are still currently original and of undetermined origin. These results highlight the links and help to trace the flows that have existed between the ancient world and Argentina. They are a particularly good example of the interest of the 2 traditional ways of multiplication which may exist for the vine, cuttings and seedlings. Some of these resources are now also highly original and rare and must be preserved.
\end{abstract}

\section{Introduction}

In most of the countries of the new world, the viticulture has started to be introduced between the $16^{\text {th }}$ century and the $18^{\text {th }}$ century. In such countries, one of the main issues to develop successfully the viticulture was to find the varieties the most adapted to these specific environmental conditions. To achieve this goal, many trials, testing and introductions of material had to be done. Thereby, different varieties according to the countries have been introduced and have allowed the development of the viticulture, sometimes by luck, but also by the work and the insight of some growers, researchers, enthusiasts or personalities. The analysis and identification of accessions from Argentina may provide a valuable testimony on the origins of the different genetic resources and varieties which have been sought and used to develop the vineyard and the grape production specificity of this country. It may also provide a special light on the mode of distribution of the grapevine varieties and the respective viticultural influences of countries from which they come from $[1,2]$.

\section{Material and methods}

54 accessions from Argentina were introduced in the Vassal collection between 1952 and 1996. They come from the old collections of INTA and from different vineyards of this country (Table 1). These accessions have been analyzed using 20 microsatellite markers according to Laucou et al [3]. The profiles obtained were compared to the profiles previously referenced in the data base of INRA with a specific query created to detect the duplicates and to identify the samples. Today the dataset represents 2722 unique profiles of Vitis vinifera varieties. When similarities between DNA profiles were detected, they were then checked at the phenotypic and ampelographic levels to confirm the identifications by morphological comparisons following the code of description proposed by OIV [4].

\section{Results}

Finally, of the 54 accessions, 43 distinct varieties have been identified after DNA genotyping and ampelography checking (Table 2).

Among them, 22 varieties correspond to old traditional wine or table grape varieties from Spain, France, Italy [5], but also from Hungary, Greece, Turkey, Syria and Lebanon. 12 varieties are probably varieties selected in Argentina or South America and are mostly descendants of Muscat of Alexandria and Mission [68] (Table 3). In this category Torrontes mendocino is an exception and for the moment none of its parents were found. 4 varieties (Moscato Cerletti, Gold, Ruby Cabernet, Yubiley) are seedlings obtained in Italy, USA and Bulgaria. 2 varieties are specific mutants (Almeria rosada, Mission mutant floral) and 3 varieties (Arvina di Petralia, Santa Paula faux, Sauvignon piccolo faux) are still original and of undetermined origin.

This is an Open Access article distributed under the terms of the Creative Commons Attribution License 4.0, which permits unrestricted use, distribution, and reproduction in any medium, provided the original work is properly cited. 
Table 1. List of the accessions introduced from Argentina in the collection of Vassal.

\begin{tabular}{|c|c|c|c|}
\hline Accession name & $\begin{array}{l}\text { Accession } \\
\text { code }\end{array}$ & $\begin{array}{l}\text { Year of } \\
\text { entry }\end{array}$ & Provenance \\
\hline Agostenga & 649Mtp6 & 1990 & Rio Negro \\
\hline Almeria rosada & 0Mtp18 & 1970 & Mendoza \\
\hline Angelina & 0Mtp28 & 1990 & Rio Negro \\
\hline Arvina di Petralia & 0Mtp42 & 1990 & Rio Negro \\
\hline Balsamina & 0Mtp74 & 1988 & Rio Negro \\
\hline Balsamina & 150Mtp28 & 1968 & Mendoza \\
\hline Bonarda & 157Mtp15 & 1968 & Mendoza \\
\hline Cereza & 2121Mtp1 & 1968 & Mendoza \\
\hline Cereza & 2121Mtp2 & 1971 & Vineyard \\
\hline César & 225Mtp6 & 1968 & Mendoza \\
\hline Criolla chica $\mathrm{n}^{\circ} 2$ & 2116Mtp1 & 1952 & Vineyard \\
\hline Criolla grande & 2118Mtp2 & 1968 & Mendoza \\
\hline Criolla mediana $\mathrm{n}^{\circ} 2$ & 2117Mtp1 & 1952 & Vineyard \\
\hline Criolla $n^{\circ} 1$ & 0Mtp276 & 1996 & Mendoza \\
\hline Criolla $\mathrm{n}^{\circ} 2=\mathrm{n}^{\circ} 125$ & 0Mtp277 & 1996 & Mendoza \\
\hline $\begin{array}{l}\text { Criolla } \mathrm{n}^{\circ} 3=\text { Criolla } \\
\text { blanca }\end{array}$ & 0Mtp278 & 1996 & Mendoza \\
\hline $\begin{array}{l}\text { Criolla } n^{\circ} 5=\text { Criolla } \\
\text { Grande }\end{array}$ & 0Mtp279 & 1996 & Mendoza \\
\hline Criolla $n^{\circ} 6$ & 0Mtp280 & 1996 & Mendoza \\
\hline Criollina San Juanina & 2118Mtp1 & 1952 & Vineyard \\
\hline Faux Trebbiano & 45Mtp7 & 1972 & San Rafael \\
\hline Folle blanche & 317Mtp12 & 1968 & Mendoza \\
\hline Gibi & 45Mtp6 & 1971 & San Rafael \\
\hline Gobernador Benegas & 1248Mtp3 & 1968 & Mendoza \\
\hline Gold & 0Mtp424 & 1990 & Rio Negro \\
\hline Graciano & 9Mtp12 & 1968 & Mendoza \\
\hline Grossa da serra & 0Mtp461 & 1990 & Rio Negro \\
\hline Jubilé & 2624Mtp1 & 1970 & Mendoza \\
\hline Malvoisie musquée & 2425Mtp3 & 1972 & San Juan \\
\hline $\begin{array}{l}\text { Moscatel negro } \\
\text { du Pérou }\end{array}$ & 2571Mtp2 & 1970 & Mendoza \\
\hline Moscatel rosado & 2120Mtp1 & 1952 & Vineyard \\
\hline Moscatel rosado & 2120Mtp3 & 1968 & Mendoza \\
\hline $\begin{array}{l}\text { Moscato Cattaratto } \\
\text { Cerletti }\end{array}$ & 715Mtp1 & 1970 & Mendoza \\
\hline Muscat d'Alexandrie & 308Mtp36 & 1971 & San Juan \\
\hline Pedro Gimenez & 0Mtp863 & 1968 & Mendoza \\
\hline Pinot gris & 458Mtp18 & 1968 & Mendoza \\
\hline Pis de chèvre rouge & 0Mtp907 & 1990 & Rio Negro \\
\hline Raboso piave & 352Mtp10 & 1968 & Mendoza \\
\hline Refosco & 2047Mtp3 & 1968 & Mendoza \\
\hline Riesling italico & 199Mtp39 & 1968 & Mendoza \\
\hline Ruby Cabernet & 2313Mtp2 & 1968 & Mendoza \\
\hline San Francisco & 2119Mtp1 & 1952 & Vineyard \\
\hline Sangioveto grosso & 1354Mtp4 & 1968 & Mendoza \\
\hline Santa Morena & 848Mtp1 & 1970 & Mendoza \\
\hline Santa Paula & 1365Mtp6 & 1970 & Mendoza \\
\hline Sauvignon & 334Mtp7 & 1971 & Mendoza \\
\hline Sauvignon (Arizu) & 334Mtp12 & 1972 & San Rafael \\
\hline Sauvignon Piccolo & 0Mtp1042 & 1990 & Rio Negro \\
\hline Sauvis & 0Mtp1044 & 1990 & Rio Negro \\
\hline Sultana & 1678Mtp6 & 1968 & Mendoza \\
\hline Torrontel riojano & $2425 \mathrm{Mtp} 2$ & 1971 & Vineyard \\
\hline Torrontes mendocino & 2421Mtp1 & 1965 & Vineyard \\
\hline Torrontes riojano & $2425 \mathrm{Mtp} 1$ & 1965 & Vineyard \\
\hline Torrontes sanjuanino & 2420Mtp1 & 1965 & Vineyard \\
\hline Uva anis & 0Mtp1157 & 1989 & Mendoza \\
\hline
\end{tabular}

Table 2. List of the varieties identified with their supposed country of origin (codes according to the ISO 3166-1 alpha 3 standard) and the corresponding accessions.

\begin{tabular}{|c|c|c|c|}
\hline $\begin{array}{l}\text { Variety } \\
\text { name }\end{array}$ & Origin & $\begin{array}{l}\text { Accession } \\
\text { name }\end{array}$ & $\begin{array}{l}\text { Accession } \\
\text { code }\end{array}$ \\
\hline Ahmar Derani & SYR & Santa Morena & 848Mtp1 \\
\hline Almeria rosada & & $\begin{array}{l}\text { Almeria } \\
\text { rosada }\end{array}$ & 0Mtp18 \\
\hline Arvina di Petralia & & $\begin{array}{l}\text { Arvina di } \\
\text { Petralia }\end{array}$ & 0Mtp42 \\
\hline $\begin{array}{l}\text { Balsamina faux } \\
\text { (collection Rio } \\
\text { Negro) }\end{array}$ & & Balsamina & 0Mtp74 \\
\hline Béquignol & FRA & Raboso piave & 352Mtp10 \\
\hline Bonamico & ITA & $\begin{array}{l}\text { Sangioveto } \\
\text { grosso }\end{array}$ & 1354Mtp4 \\
\hline Canari & FRA & Pinot gris & 458Mtp18 \\
\hline \multirow{2}{*}{ Cereza } & & Cereza & 2121Mtp1 \\
\hline & & Cereza & 2121Mtp2 \\
\hline César & FRA & César & 225Mtp6 \\
\hline Chardonnay & FRA & $\begin{array}{l}\text { Riesling ital- } \\
\text { ico }\end{array}$ & 199Mtp39 \\
\hline Corbeau & FRA & Bonarda & 157Mtp15 \\
\hline \multirow{2}{*}{ Criolla 5} & & $\begin{array}{l}\text { Criolla } \mathrm{n}^{\circ} 2= \\
\mathrm{n}^{\circ} 125\end{array}$ & 0Mtp277 \\
\hline & & $\begin{array}{l}\text { Criolla } \mathrm{n}^{\circ} 5= \\
\text { Criolla Grande }\end{array}$ & 0Mtp279 \\
\hline Criolla blanca & & $\begin{array}{l}\text { Criolla } n^{\circ} 3= \\
\text { Criolla blanca }\end{array}$ & 0Mtp278 \\
\hline \multirow{3}{*}{$\begin{array}{l}\text { Criolla grande } \\
\text { sanjuanina }\end{array}$} & & Criolla $n^{\circ} 1$ & 0Mtp276 \\
\hline & & $\begin{array}{l}\text { Criollina San } \\
\text { Juanina }\end{array}$ & 2118Mtp1 \\
\hline & & $\begin{array}{l}\text { Criolla } \\
\text { grande }\end{array}$ & $2118 \mathrm{Mtp} 2$ \\
\hline Folle blanche & FRA & Folle blanche & 317Mtp12 \\
\hline \multirow{2}{*}{ Gibi } & \multirow{2}{*}{ ESP } & Gibi & 45Mtp6 \\
\hline & & $\begin{array}{l}\text { Faux } \\
\text { Trebbiano }\end{array}$ & $45 \mathrm{Mtp} 7$ \\
\hline Giro sardo & ITA & Sauvis & 0Mtp1044 \\
\hline Gold & USA & Gold & 0Mtp424 \\
\hline $\begin{array}{l}\text { Kecskecsecsu } \\
\text { roszas piros }\end{array}$ & HUN & $\begin{array}{l}\text { Pis de chèvre } \\
\text { rouge }\end{array}$ & 0Mtp907 \\
\hline Kichmich rond & TUR & Sultana & 1678Mtp6 \\
\hline $\begin{array}{l}\text { Lambrusco } \\
\text { Maestri }\end{array}$ & ITA & Refosco & 2047Mtp3 \\
\hline $\begin{array}{l}\text { Madeleine } \\
\text { Salomon = } \\
\text { Agostenga blanc }\end{array}$ & ITA & Agostenga & 649Mtp6 \\
\hline \multirow{2}{*}{ Mission } & \multirow{2}{*}{ ESP } & Criolla $n^{\circ} 6$ & 0Mtp280 \\
\hline & & $\begin{array}{l}\text { Moscatel } \\
\text { negro du Pérou }\end{array}$ & 2571Mtp2 \\
\hline $\begin{array}{l}\text { Mission mutant } \\
\text { floral }\end{array}$ & & $\begin{array}{l}\text { Criolla chica } \\
n^{\circ} 2\end{array}$ & 2116Mtp1 \\
\hline Morrastel & ESP & Graciano & 9Mtp12 \\
\hline \multirow{2}{*}{ Moscatel rosado } & & $\begin{array}{l}\text { Moscatel } \\
\text { rosado }\end{array}$ & 2120Mtp1 \\
\hline & & $\begin{array}{l}\text { Moscatel } \\
\text { rosado }\end{array}$ & 2120Mtp3 \\
\hline
\end{tabular}


Table 2. Continued.

\begin{tabular}{|c|c|c|c|}
\hline $\begin{array}{l}\text { Variety } \\
\text { name }\end{array}$ & Origin & $\begin{array}{l}\text { Accession } \\
\text { name }\end{array}$ & $\begin{array}{l}\text { Accession } \\
\text { code }\end{array}$ \\
\hline Moscato Cerletti & ITA & $\begin{array}{l}\text { Moscato } \\
\text { Cattaratto } \\
\text { Cerletti }\end{array}$ & $715 \mathrm{Mtp} 1$ \\
\hline $\begin{array}{l}\text { Muscat } \\
\text { d'Alexandrie }\end{array}$ & GRC & $\begin{array}{l}\text { Muscat } \\
\text { d'Alexandrie }\end{array}$ & 308Mtp36 \\
\hline Nielluccio & ITA & Angelina & 0Mtp28 \\
\hline $\begin{array}{l}\text { Pascale }=\text { Pascale } \\
\text { di Cagliari }\end{array}$ & ITA & $\begin{array}{l}\text { Grossa da } \\
\text { serra }\end{array}$ & 0Mtp461 \\
\hline $\begin{array}{l}\text { Pedro Gimenez } \\
\text { faux }\end{array}$ & & $\begin{array}{l}\text { Pedro } \\
\text { Gimenez }\end{array}$ & 0Mtp863 \\
\hline \multirow{2}{*}{ Rosa del Peru } & & $\begin{array}{l}\text { Criolla medi- } \\
\text { ana } n^{\circ} 2\end{array}$ & 2117Mtp1 \\
\hline & & $\begin{array}{l}\text { San } \\
\text { Francisco }\end{array}$ & 2119Mtp1 \\
\hline Ruby Cabernet & USA & $\begin{array}{l}\text { Ruby } \\
\text { Cabernet }\end{array}$ & 2313Mtp2 \\
\hline $\begin{array}{l}\text { Santa Paula faux } \\
\text { (collection } \\
\text { Mendoza) }\end{array}$ & & Santa Paula & 1365Mtp6 \\
\hline $\begin{array}{l}\text { Sauvignon } \\
\text { piccolo faux }\end{array}$ & & $\begin{array}{l}\text { Sauvignon } \\
\text { Piccolo }\end{array}$ & 0Mtp1042 \\
\hline \multirow[t]{2}{*}{ Sauvignonasse } & \multirow[t]{2}{*}{ FRA } & $\begin{array}{l}\text { Sauvignon } \\
\text { (Arizu) }\end{array}$ & 334Mtp12 \\
\hline & & Sauvignon & 334Mtp7 \\
\hline Syrah & FRA & Balsamina & 150Mtp28 \\
\hline $\begin{array}{l}\text { Torrontès } \\
\text { mendocino }\end{array}$ & & $\begin{array}{l}\text { Torrontes } \\
\text { mendocino }\end{array}$ & 2421Mtp1 \\
\hline \multirow[t]{3}{*}{ Torrontès riojano } & & $\begin{array}{l}\text { Torrontes } \\
\text { riojano }\end{array}$ & 2425Mtp1 \\
\hline & & $\begin{array}{l}\text { Torrontel } \\
\text { riojano }\end{array}$ & 2425Mtp2 \\
\hline & & $\begin{array}{l}\text { Malvoisie } \\
\text { musquée }\end{array}$ & 2425Mtp3 \\
\hline $\begin{array}{l}\text { Torrontès } \\
\text { sanjuanino }\end{array}$ & & $\begin{array}{l}\text { Torrontes } \\
\text { sanjuanino }\end{array}$ & 2420Mtp1 \\
\hline Uva anis & & Uva anis & 0Mtp1157 \\
\hline Yubiley & BGR & Jubilé & 2624Mtp1 \\
\hline Zeini abiad & LBN & $\begin{array}{l}\text { Gobernador } \\
\text { Benegas }\end{array}$ & $1248 \mathrm{Mtp} 3$ \\
\hline
\end{tabular}

Table 3. Parentage of the varieties selected in South America according to the results obtained by [6-8].

\begin{tabular}{|l|l|l|}
\hline Variety name & Parent 1 & Parent 2 \\
\hline $\begin{array}{l}\text { Balsamina faux } \\
\text { (collection Rio Negro) }\end{array}$ & Cot & $\begin{array}{l}\text { Torrontes } \\
\text { mendocino }\end{array}$ \\
\hline Cereza & Muscat d'Alexandrie & Mission \\
\hline Criolla 5 & Muscat d'Alexandrie & Mission \\
\hline Criolla blanca & Muscat d'Alexandrie & Mission \\
\hline $\begin{array}{l}\text { Criolla grande } \\
\text { sanjuanina }\end{array}$ & Muscat d'Alexandrie & Mission \\
\hline Pedro Gimenez faux & Muscat d'Alexandrie & Mission \\
\hline Rosa del Peru & Muscat d'Alexandrie & Mission \\
\hline Torrontès riojano & Muscat d'Alexandrie & Mission \\
\hline Torrontès sanjuanino & Muscat d'Alexandrie & Mission \\
\hline Uva anis & Muscat d'Alexandrie & Mission \\
\hline Moscatel rosado & Muscat d'Alexandrie & $?$ \\
\hline
\end{tabular}

\section{Discussion and conclusion}

These results highlight the links and help to trace the flow and the relationships that have existed between the ancient world and Argentina, which led to the specificity of the present Argentina vineyard. They are a particularly good example of the interest of the 2 traditional ways of multiplication which may exist for the vine: cuttings to introduced already existing and possibly interesting varieties and seedlings to select new genotypes adapted to the specific environmental conditions of the country. Some of these resources are now also highly original and relatively rare that is why some effort must be done to preserve them by the best possible way.

\section{References}

[1] G. Cipriani, A. Spadotto, I. Jurman, G. Di Gaspero, M. Crespan, S. Meneghetti, E. Frare, R. Vignani, M. Cresti, M. Morgante, M. Pezzotti, E. Pe, A. Policriti, R. Testolin, Theor Appl Genet 121 (2010)

[2] R. Bacilieri, T. Lacombe, L. Le Cunff, M. Di VecchiStaraz, V. Laucou, B. Genna, J.P. Péros, P. This, J.M. Boursiquot, BMC Plant Biology 13:25 (2013)

[3] V. Laucou, T. Lacombe, F. Dechesne, R. Siret, J.P. Bruno, M. Dessup, T. Dessup, P. Ortigosa, P. Parra, C. Roux, S. Santoni, D. Varès, J.P. Péros, J.M. Boursiquot, P. This, Theor Appl Genet 122 (2011)

[4] OIV, Second edition of the OIV descriptor list for grape varieties and Vitis species (2009)

[5] L. Martinez, P. Cavagnaro, J.M. Boursiquot, C. Agüero, Am J Enol Vitic 59:3 (2008)

[6] C. Agüero, J. Rodriguez, L. Martinez, G. Dangl, C. Meredith, Am J Enol Vitic 54:3 (2003)

[7] A.M. Tapia, J. Cabezas, F. Cabello, T. Lacombe, J.M. Martinez-Zapater, P. Hinrichsen, M. Cervera, Am J Enol Vitic 58:2 (2007)

[8] T. Lacombe, J.M. Boursiquot, V. Laucou, M. Di Vecchi-Staraz, J.P. Péros, P. This, Theor Appl Genet 124 (2013) 